UNESUM-Ciencias: Revista Científica Multidisciplinaria ISSN 2602-8166

Publicación cuatrimestral. Vol. 5, No. 4 (Número Especial), Año 2021.Pág. 169-184

PROPUESTA METODOLÓGICA E IMPLEMENTACIÓN DE UNA RED LAN

\title{
PROPUESTA METODOLÓGICA E IMPLEMENTACIÓN DE UNA RED LAN PARA EL INSTITUTO DE MEDICINA DEPORTIVA
}

AUTORES: Ana Margarita Sardiñas Ramírez ${ }^{1}$

Omar Mar Cornelio ${ }^{2}$

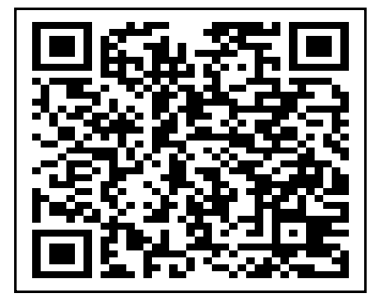

\section{DIRECCIÓN PARA CORRESPONDENCIA: ana.sardinas@inder.gob.cu}

Fecha de recepción: 10/012/2020

Fecha de aceptación: 20/06/2021

\section{RESUMEN}

La implementación de una red LAN permite facilitar la administración a través de las nuevas tecnologías, sean capaces de comunicarse entre sí y acceder a los datos, servicios de procesamiento, aplicaciones y otros recursos, lo cual reduce el costo de funcionamiento interno, tanto a nivel económico como de tiempos de respuesta al usuario. Debido a los problemas planteados, y conociendo las ventajas que ofrecen las redes de sistemas computacionales, se propone una metodología para el análisis y diseño de una red LAN, la cual pretende mejorar la competitividad, reducción de costos operacionales, aumentando las ganancias en tiempo y dinero. Las redes están siempre en crecimiento continuo se podría garantizar con procedimientos como la estrategia del servicio unido con gestión de la demanda, en la cual se debe definir cuáles son los requisitos y necesidades. Se deben definir e identificar cuáles son los objetivos que son factor clave para el crecimiento. Permite establecer lineamientos de control de servicios para implementarlos en el análisis y diseño de una red que genera una mejor gestión sobre implementación de nuevas versiones de hardware y/o software, monitoreo, recolección, registro y análisis de datos de una red y mejora en gran medida la calidad del servicio.

PALABRAS CLAVE: LAN; interconexión; redes de computadoras; implementación

\section{METHODOLOGICAL PROPOSAL AND IMPLEMENTATION OF A LAN FOR THE INSTITUTE OF SPORTS MEDICINE}

\section{ABSTRACT}

The implementation of a LAN allows to facilitate administration through new technologies, being able to communicate with each other and access data, processing services, applications and other

\footnotetext{
${ }^{1}$ Ingeniera en Ciencias Informáticas, Estudiante de la Maestría en Informática Médica Aplicada UCI, Jefa del Departamento de Informática del Instituto de Medicina Deportiva, La Habana, Cuba. E-mail: ana.sardinas@inder.gob.cu

${ }^{2}$ Doctor en Ciencias Técnicas, Centro de Estudios de la Matemática Computacional, Universidad de las Ciencias Informáticas., La Habana, Cuba. E-mail: omarmar@uci.cu
} 
resources, which reduces the cost of internal operation, both at the economic as well as response times to the user. Due to the problems raised, and knowing the advantages offered by computer systems networks, a methodology is proposed for the analysis and design of a LAN, which aims to improve competitiveness, reduce operational costs, and increase profits in time and money. Networks are always in continuous growth, it could be guaranteed with procedures such as the service strategy combined with demand management, in which the requirements and needs must be defined. The objectives that are a key factor for growth must be defined and identified. It allows to establish service control guidelines to implement them in the analysis and design of a network that generates a better management on the implementation of new versions of hardware and / or software, monitoring, collection, recording and analysis of data from a network and greatly improves measure the quality of service.

KEYWORDS: LAN; Computer Networks; interconnection; implementation

\section{INTRODUCCIÓN}

La necesidad de crear una metodología de redes basada en servicios para dar un enfoque diferente, que permita realizar el análisis y diseño de una red tomando consideraciones necesarias para el crecimiento continuo y a su vez implementar estrategias de servicio para el control, manejo y atención de incidentes que se presentan en la red.

Se propone una metodología con los pasos básicos y necesarios para realizar un análisis y diseño de redes, principalmente se establecen las pautas requeridas para el levantamiento de información, para esto se especifican las mejores prácticas y se saca el mejor provecho en la detección de las necesidades del cliente e identificación de recursos del sistema, posterior a esto se establece una gestión de la capacidad para determinar la capacidad del diseño a implementar, posterior a esto se establecen los lineamientos necesarios para el diseño de red considerando factores influyentes de la tecnología encaminándose a los objetivos del IMD y la proyección para crecimiento a futuro, se establece la forma en cómo se debe realizar la administración de red, definición de roles para la red y consideraciones a tener en cuenta para el manejo y control de incidentes, a la seguridad y como se debe controlar, la experiencia en redes genera un valor agregado para la identificación de necesidades, análisis de componentes de una red, diseño de la solución y definición de políticas de seguridad (Mar-Cornelio et al., 2019), (Caycho-Rodríguez et al., 2021)

Para el levantamiento de información en redes se identifican las necesidades con los usuarios por medio de entrevistas y estudio del medio, se define en la gestión de servicios que "El cliente centra su interés en el valor de uso y prefiere mantenerse al margen de los detalles técnicos y de estructura. El "principio de encapsulación” se basa en ocultar al cliente lo que no necesita y en mostrarle lo que le resulta útil y valioso (Hernán-García et al., 2021), (Mar et al., 2019). En parte el concepto de levantamiento con los usuarios es correcto pero se debe especificar lo que resultara para el cliente como un medio para entregar valor, facilitando los resultados que los usuarios quieren conseguir sin asumir costes o riesgos específicos.

\section{DESARROLLO}

El diseño de una red debe ser eficaz y eficiente buscando el equilibrio entre ganancia y costo, este inicia con la demanda de nuevos requerimientos y se debe involucrar a todo el personal del área

170 UNESUM-Ciencias.Publicación cuatrimestral. Vol. 5, Año 2021, No. 4 (Número Especial), 
UNESUM-Ciencias: Revista Científica Multidisciplinaria ISSN 2602-8166

Publicación cuatrimestral. Vol. 5, No. 4 (Número Especial), Año 2021.Pág. 169-184 PROPUESTA METODOLÓGICA E IMPLEMENTACIÓN DE UNA RED LAN

de tecnología, es de esta manera que se inicia el proceso metodológico para análisis y diseño de red (Bustos-Claro et al., 2021), (Mar et al., 2015). Se debe tener en cuenta que no existe un diseño estándar del que se pueda tomar referencia, si no que existen lineamientos básicos para cada red, se busca entonces crear una y auditoria de una red. "Lo primero que se debe definir son los objetivos a los cuales apunta una red de datos, se deben tener en cuenta los siguientes 4 requerimientos importantes” (Ávila-Rodríguez et al., 2021), (Cornelio, 2016):

Funcionalidad: una red debe ser funcional, debe permitir que los usuarios de red cumplan con los requisitos de trabajo, debe proveer conectividad entre los usuarios y aplicaciones a tiempos de respuesta razonables (Martinez-Perez et al., 2021), (Mar Cornelio et al., 2016).

Escalabilidad: Todas las redes deben ser capaces de crecer continuamente y abordar las nuevas tecnologías minimizando los costes de implementación, esto es uno de los requerimientos que más fácil se deja de tener en cuenta en el análisis de la red, en la mayoría de los casos no se prevé el crecimiento de estructura.

Adaptabilidad: Se debe realizar el diseño de redes teniendo en cuenta tecnologías futuras y no se debe limitar la red para la implementación de estas nuevas tecnologías mientras se puedan adquirir.

Administración: El diseño de la red debe resultar de fácil manejo para el monitoreo, administración y control de incidencias. Respecto al diseño físico de la red se deben identificar como primera medida la colocación física y función de los servidores, esto se debe a que existen servidores que están enfocados a proveer servicios tales como compartir archivos, impresión y comunicación así como servicios de aplicación, el no ubicar adecuadamente estos dispositivos de red puede incrementar el uso. Dispositivos para interconexión y así maximizar el costo de implementación, mantenimiento y en caso extremo mayor número de incidencias (Barba et al., 2018), (Mar Cornelio et al., 2016). 


\section{Metodología del diseño LAN}

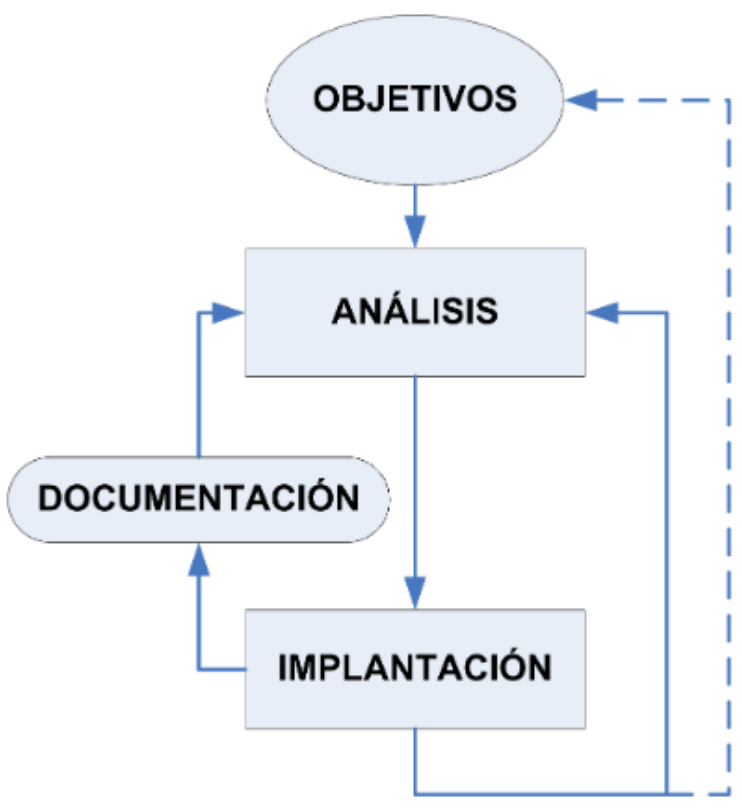

Figura.1 Metodología del diseño LAN

El sistema informático del Instituto de Medicina del Deporte (IMD) se encuentra sustentado sobre una Red Local con topología Árbol y cableado estructurado categoría 5e, abarcando las áreas situadas en la planta baja y primer piso del Edificio (Enríquez et al., 2019), (Cornelio \& Gulín, 2018).

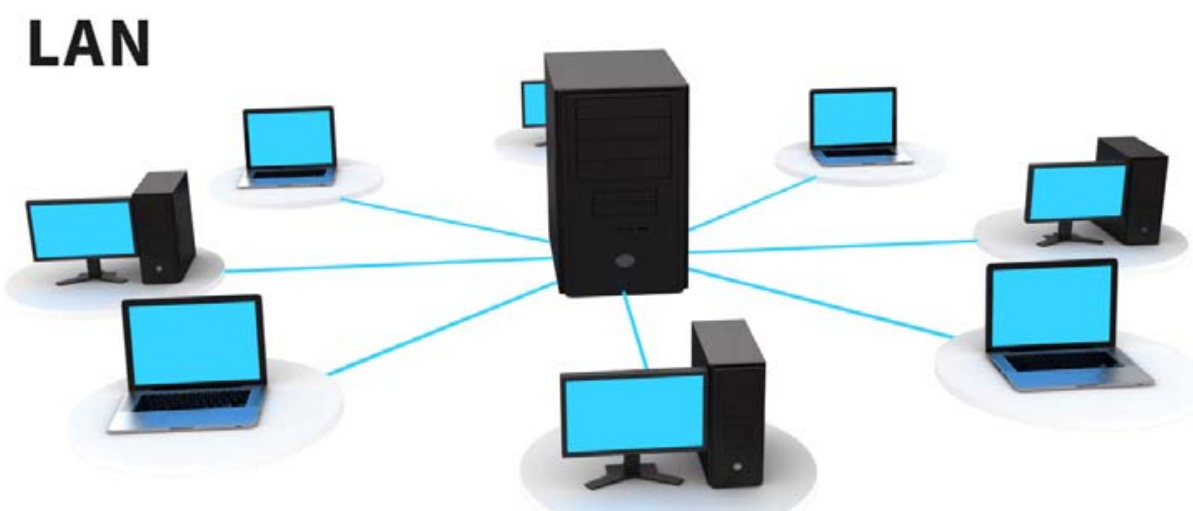

Figura.2 Diseño LAN

172 UNESUM-Ciencias.Publicación cuatrimestral. Vol. 5, Año 2021, No. 4 (Número Especial), 
UNESUM-Ciencias: Revista Científica Multidisciplinaria ISSN 2602-8166

Publicación cuatrimestral. Vol. 5, No. 4 (Número Especial), Año 2021.Pág. 169-184 PROPUESTA METODOLÓGICA E IMPLEMENTACIÓN DE UNA RED LAN

Se cuenta además con cinco Servidores que se encargan de administrar el funcionamiento del sistema, los servicios que se brindan la seguridad y protección del mismo y 57estaciones de trabajo, estando todas conectadas en Red.

LosServidoresutilizan como Sistemas Operativos Windows Server 2003, 2008, 2010, Windows Server 2012, PROMOX 4.0 y en las estaciones de trabajo están instalados los Sistemas Operativos Windows XP/7/8/10.

Los Servidores tienen las siguientes funciones:

- $\quad$ Proxy Web y Firewall

- $\quad$ Controlador de Dominio, FTP y Correo Electrónico

- $\quad$ Sistema Contable (Versat)

- $\quad$ Clientes Ligeros

- $\quad$ PROMOX (Controlador de Dominio, Mensajería Instantánea)

Los servicios implementados en la red son de navegación, correo electrónico, y transferencia de ficheros. La navegación y el correo electrónico tienen alcance nacional o internacional en dependencia de lo aprobado para cada usuario a partir de sus necesidades.

Aplicaciones en explotación:

El Instituto de Medicina del Deporte desarrolla y garantiza sus actividades médicas, docentes, investigativas y administrativas con el siguiente soporte de software:

- Sistemas Operativos

- Paquetes Ofimáticos

- Paquetes Estadísticos.

- Editores de páginas Web.

- Software de comunicaciones

- Software de administración de redes locales.

- Software utilitario de uso general.

- Software de aplicación.

\section{Medios de Transmisión:}


El cableado de la red está soportado por Cable UTP Categoría 5e protegido con canaletas y la red local opera a una velocidad de 100/1000 Mbps con topología Árbol.

Las estaciones de trabajo se agrupan por áreas y pisos a partir de conmutadores (Switchs) Capa 2 y así poder compartir todos los recursos con que cuenta la misma.

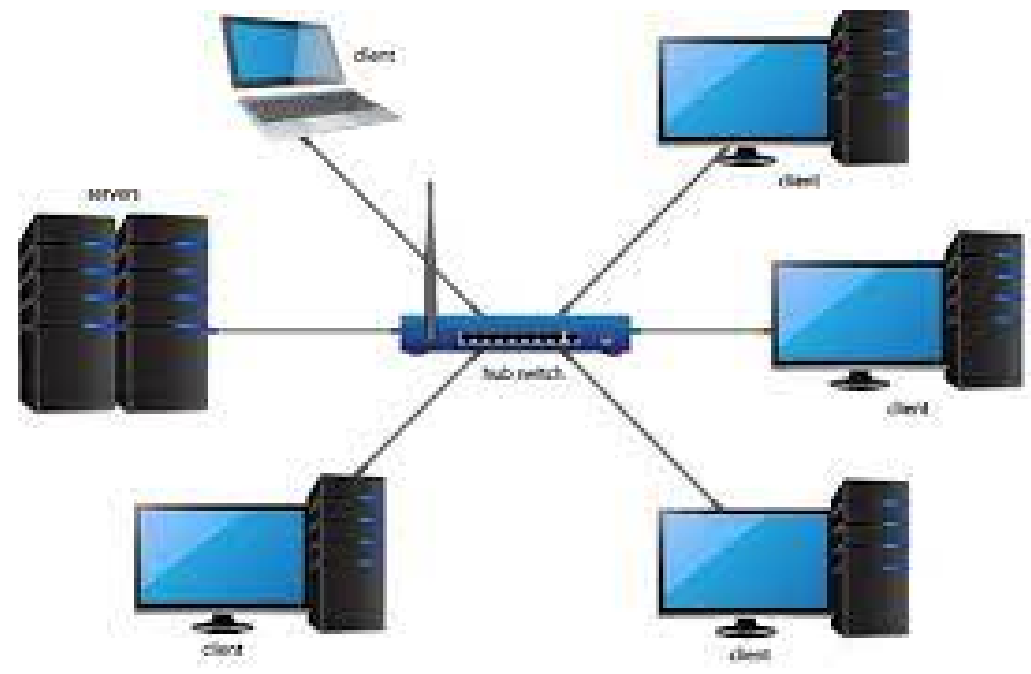

Figura.3 Diagrama de red de área local

La conexión con el exterior se realiza disponiendo de 1 línea arrendada para la transmisión de datos, conectada con ETECSA a una velocidad de 2 Mbps. A través de esta accedemos a nuestro proveedor de servicios de navegación nacional, INFOMED (Red Telemática de Salud de Cuba), que es el Nodo principal de comunicaciones del Sistema Nacional de Salud.El segundo enlace es utilizando fibra óptica a 10 Mbps es a través de INDERED, que es la Red Nacional de Computadoras del Instituto Nacional de Deportes, Educación Física y Recreación (INDER), el cual nos provee de acceso a INTERNET.

174 UNESUM-Ciencias.Publicación cuatrimestral. Vol. 5, Año 2021, No. 4 (Número Especial), 


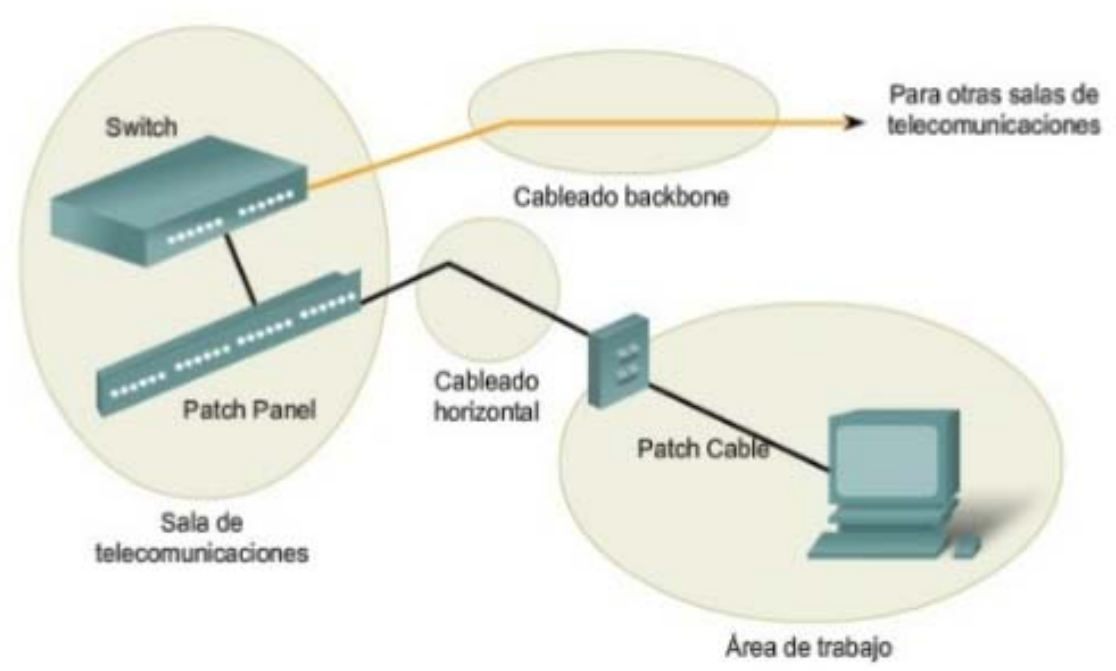

Figura.4 Áreas de cableado LAN

Mediante la conexión con INFOMED se establece el acceso de navegación nacional, por un número IP asignado con tal propósito. Este número IP se le asignó a la puerta WAN del Router TP-LINK TD, mientras que la puerta LAN del mismo se conecta a una de las 3 interfaces de red que tiene el Servidor Proxy (TMG). La conexión al INDER se realiza por la segunda interface de red que está conectada a otro router One Access, el cual tiene en la puerta WAN una IP asignada por el INDER. 


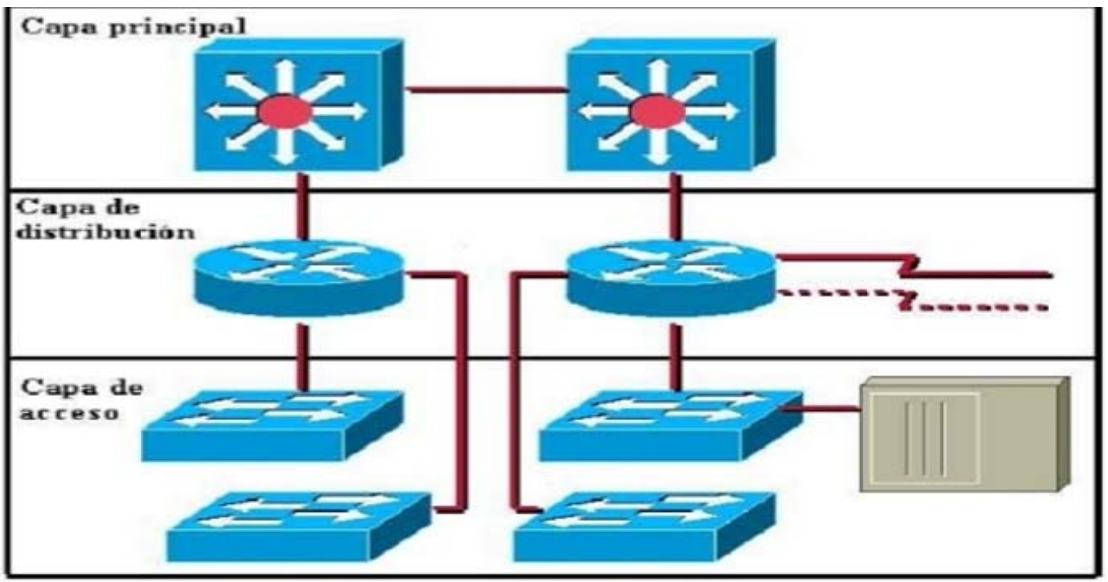

Figura.5 Capa principal, de distribución y de acceso

La tercera interface de red del servidor proxy está conectada a los switchs, delque están colgados el resto de los servidores y las estaciones de trabajo de la red local. Tanto los servidores como las estaciones de trabajo cuentan con número IP ficticio.

El intercambio de información tanto interna como externa se realiza básicamente a través del correo electrónico.

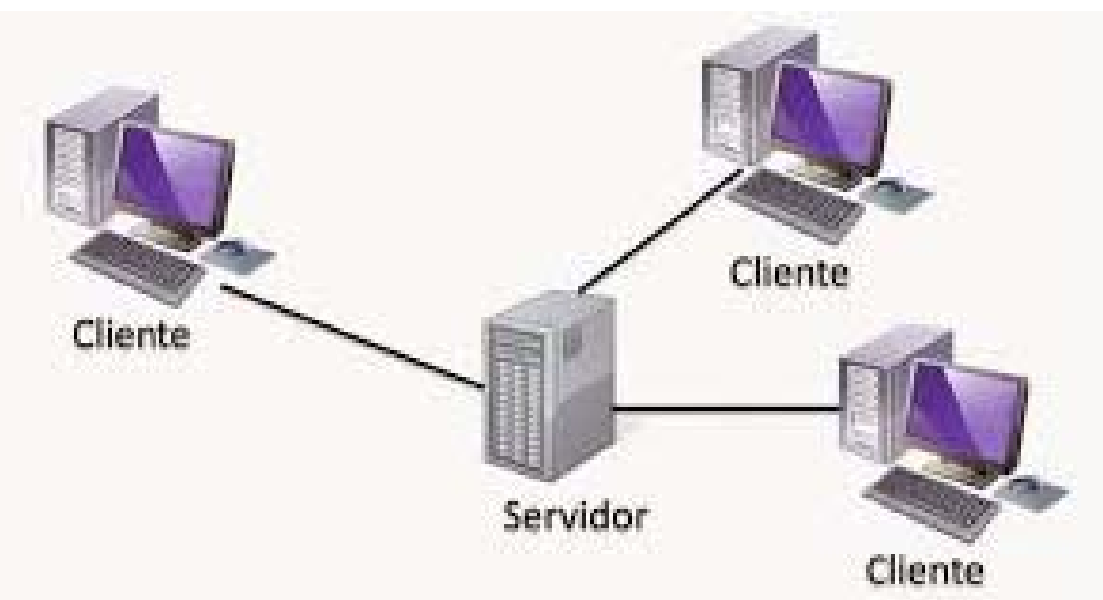

Figura.6 Servidor de correo y clientes

176 UNESUM-Ciencias.Publicación cuatrimestral. Vol. 5, Año 2021, No. 4 (Número Especial), 
UNESUM-Ciencias: Revista Científica Multidisciplinaria ISSN 2602-8166

Publicación cuatrimestral. Vol. 5, No. 4 (Número Especial), Año 2021.Pág. 169-184 PROPUESTA METODOLÓGICA E IMPLEMENTACIÓN DE UNA RED LAN

El Instituto tiene buenas condiciones constructivas, adecuadas tanto para la protección como para la preservación de los equipos y la posibilidad de visibilidad de las pantallas desde el exterior es prácticamente nula.

El personal que opera los equipos posee los conocimientos y la preparación necesaria para su empleo y en su mayoría tienen calificación, médicos, enfermeras, psicólogos, fisioterapeutas, especialistas.

\section{Impacto de la Informatización y principales líneas de trabajo en la institución:}

El impacto que ha tenido el uso de la Informática y la Telemática ha sido decisivo en el cumplimiento de las actividades del IMD, constituyendo la plataforma informática un apoyo esencial para el desempeño laboral.

Para los especialistas del Centro: Personal médico, docentes e investigadores y técnicos, el desarrollo informático ha repercutido en una mayor calidad y productividad de sus tareas. Los servicios mencionados en el punto anterior son utilizados en los diferentes departamentos Docentes y no Docentes y Grupos de trabajo, facilitando la ejecución de sus principales líneas de trabajo, las cuales se relacionan a continuación:

\section{Principales líneas de trabajo en la docencia:}

a. Formar Profesionales en la Medicina del Deporte con una sólida preparación científico-técnica y político-ideológica desde una perspectiva integradora docente, asistencial e investigativa que permita dar solución a los problemas de la medicina del deporte y sus ciencias aplicadas, con los niveles de desempeño y competencia que necesita nuestra sociedad.

b. Impartir cursos de Postgrado para continuar la formación continua de Profesionales de la medicina del deporte, Especialistas, Máster y Doctores en Ciencias cubanos y extranjeros con la más alta calidad humana.

c. Tutorar Tesis a Residentes y Especialistas.

d. Otras tareas de capacitación orientadas

\section{Principales líneas de trabajo en la investigación:}

a. Educación y Control antidopaje 

b. Promoción y salud
c. Medicina Natural y Tradicional
d. Ciencias aplicadas al deporte
e. Informática Educativa

Principales líneas de trabajo de los Servicios Informáticos:

a. Garantizar la conectividad del IMD con nuestro proveedor de servicios Infomed e INDERED.

b. Diseñar y desarrollar herramientas que garanticen el óptimo funcionamiento de la administración de la red.

c. Garantizar la administración de los sitios tanto de la intranet como de internet.

d. Trazar políticas, reglamentos y directrices generales para el uso y explotación de la red del Centro.

e. Diseñar y aplicar estrategias para iniciar el proceso de migración hacia el software libre en el IMD

\section{Resultado del Análisis de Riesgos}

El sistema informático del Instituto de Medicina del Deporte es de suma importancia, pues el mismo procesa y mantiene la información que se necesita para operar interna y externamente, por lo que su deterioro provocaría una limitación en tiempo real del procesamiento de la información.

Las tablas siguientes muestran la relación de los activos o bienes informáticos del centro, identificados según el área a la que pertenecen, valorando su importancia a partir del papel que juegan dentro del mismo.

\section{Estudio de vulnerabilidad de los Sistemas Informáticos}

\begin{tabular}{|c|l|}
\hline No. & \multicolumn{1}{|c|}{ DESCRIPCIÓN } \\
\hline 1 & Oficina del Director \\
\hline 2 & Oficina de las Secretarias de la Dirección \\
\hline 3 & Subdirección Primera \\
\hline 4 & Oficina Asesor \\
\hline
\end{tabular}

178 UNESUM-Ciencias.Publicación cuatrimestral. Vol. 5, Año 2021, No. 4 (Número Especial), 
UNESUM-Ciencias: Revista Científica Multidisciplinaria ISSN 2602-8166

Publicación cuatrimestral. Vol. 5, No. 4 (Número Especial), Año 2021.Pág. 169-184 PROPUESTA METODOLÓGICA E IMPLEMENTACIÓN DE UNA RED LAN

\begin{tabular}{|c|c|}
\hline 5 & Subdirección de Aseguramiento \\
\hline 6 & Subdirección de Docencia e Investigaciones \\
\hline 7 & Subdirección de Control Médico \\
\hline 8 & Subdirección de Asistencia Médica \\
\hline 9 & Subdirección de Economía \\
\hline 10 & Subdirección de Psicología \\
\hline 11 & Departamento de Recursos Humanos \\
\hline 12 & Oficina de Cuadros \\
\hline 13 & Biblioteca \\
\hline 14 & Departamento de Enfermería \\
\hline 15 & Oficina de Composición corporal \\
\hline 16 & Oficina de Neurofisiología \\
\hline 17 & Oficina de Fisiología Cardiovascular \\
\hline 18 & Departamento de Estomatología \\
\hline 19 & Subdirección de Medicina Física y Rehabilitación \\
\hline 20 & Departamento de Informática \\
\hline 21 & Salón de Profesores \\
\hline 22 & Laboratorio Central \\
\hline 23 & Aula 1 \\
\hline 24 & Aula Internacional \\
\hline 25 & Oficina Control Antidoping \\
\hline
\end{tabular}

\begin{tabular}{|c|c|c|}
\hline \multicolumn{3}{|c|}{ TIPOS DE ACTIVOS } \\
\hline ABR & DESCRIPCIÓN & TOTALES \\
\hline HW & Hardware & 95 \\
\hline SW & Software & 50 \\
\hline DT & Datos & 18 \\
\hline \multicolumn{2}{|c|}{ TOTAL DE ACTIVOS IDENTIFICADOS } & 163 \\
\hline
\end{tabular}




\section{Medios Técnicos}

Sistemas Operativos y nivel de seguridad instalado:

Los Servidores utilizan como Sistemas Operativos Windows Server Enterprise Edition con Service Pack 2 y Windows 2012 R2.

Las estaciones de trabajo utilizan como Sistemas Operativos Windows XP con Service Pack 3, Windows 7 con Service Pack 2, Windows 8.1 y Windows 10.

Las estaciones de trabajo con Windows XP/7/8.1/10 están unidas al Controlador de Dominio de la red con Active Directory, lo que permite que cada usuario entre con su login y password, y haga uso solamente de los privilegios y servicios dados por el Administrador de la red.

En nuestra red contamos con dos controladores de Dominio con Catálogo Global los que contienen la Base de datos de todos los objetos de la red. Los costos son gastos de alto nivel, como hardware, software, personal, administración, ubicaciones físicas y servicios externos.

Los tipos de costos se subdividen a su vez en elementos de coste. Elementos de costo del hardware, por ejemplo, serían servidores, computadoras, switchers, impresoras, etc.

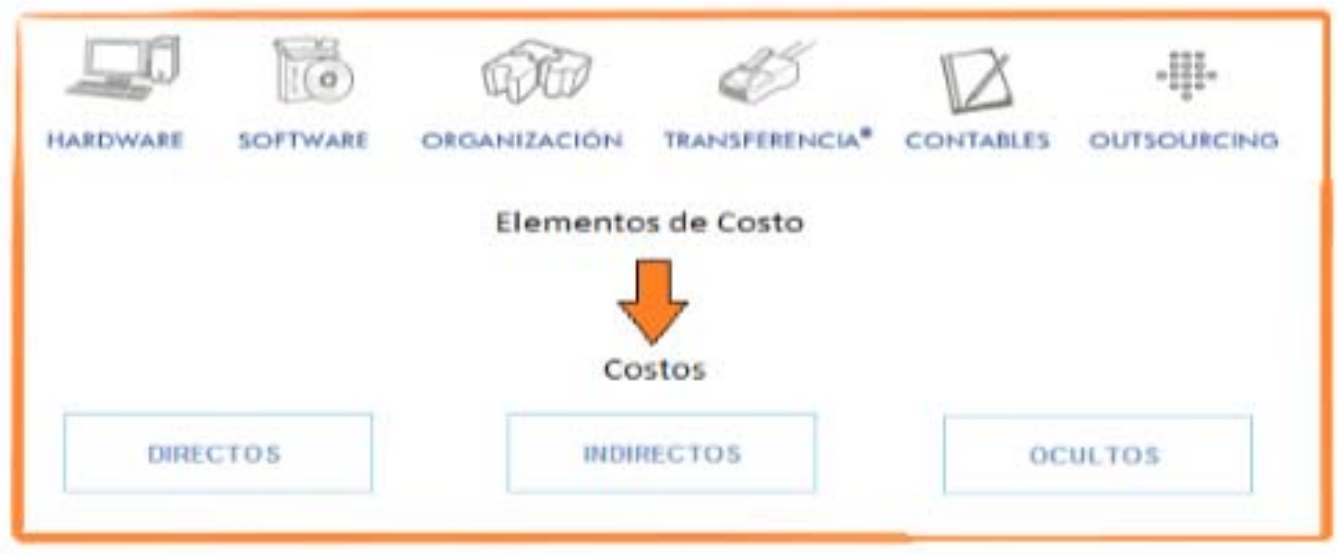

Figura. 7 Elementos de costo

180 UNESUM-Ciencias.Publicación cuatrimestral. Vol. 5, Año 2021, No. 4 (Número Especial), 
UNESUM-Ciencias: Revista Científica Multidisciplinaria ISSN 2602-8166

Publicación cuatrimestral. Vol. 5, No. 4 (Número Especial), Año 2021.Pág. 169-184 PROPUESTA METODOLÓGICA E IMPLEMENTACIÓN DE UNA RED LAN

Tipos de redes utilizadas y topología de las mismas.

El sistema informático del Instituto de Medicina del Deporte está soportado en medios informáticos que incluyen 5Servidores y 50 estaciones de trabajo, conectadas a una Red Local con topología de Árbol que abarca las áreas situadas en la planta baja y primer piso del Edificio y está conectada a la Red Telemática de Salud de Cuba Infomed a través de una línea arrendada ADSL a una velocidad de transmisión de 2 Mbps y a la Red Nacional de Computadoras del Instituto Nacional de Deportes, Educación Física y Recreación (INDER) a través de un enlace óptico con velocidad de transmisión de 10 Mbps.

\section{Servicios que se ofrecen:}

\section{Correo Electrónico:}

A través de este servicio pueden asignarse cuentas de correos a los usuarios del Instituto de Medicina del Deporte. Para ello se capacitó y adiestró a la mayoría de los usuarios, especialistas, fisioterapeutas, médicos, enfermeras y técnicos en su manejo, ya que es la base principal de intercambio de información con las demás dependencias de la Universidad y con otras entidades nacionales e internacionales.

La mensajería tiene alcance nacional e internacional, existen 147 buzones de médicos, profesores, técnicos y especialistas con cuentas para el dominio “imd.inder.cu” y 334 cuentas en el dominio “infomed.sld.cu”, de ellas ninguna con acceso telefónico. Por su parte existen 103 cuentas del dominio “inder.cu”, todas con acceso telefónico.

\section{Web:}

A través de este servicio los usuarios autorizados pueden acceder a las páginas web nacionales e internacionales según sus privilegios de acceso.

Todos los usuarios autorizados pueden consultar una enorme fuente de información disponible en internet.

Por medio de este servicio se actualiza el sitio Web del Instituto de Medicina del Deporte.

\section{FTP:}

Se cuenta con un servidor FTP para servicio interno del IMD donde se almacenan: 
- $\quad$ Actualizaciones de los antivirus

- $\quad$ Instalaciones de Sistemas Operativos

- Instalaciones de Aplicaciones (WEB, Multimedia, Paquetes Ofimáticos, Antivirus, Herramientas de Recuperación, etc.)

- $\quad$ Utilitarios

- $\quad$ Documentos, Modelos y archivos públicos para el personal del IMD

- $\quad$ Literatura Docente, Manuales, Tutoriales, Revistas TICs y Literatura Universal

- $\quad$ Multimedias educativas

\section{Barreras de protección y su arquitectura}

Los locales están distribuidos en dos pisos y tienen buena estructura arquitectónica de consistencia sólida. El local del nodo de comunicaciones cumple con los requisitos de arquitectura que se exigen para este fin. Empleo de Firewall, de Hosts Bastiones, Sistema Proxy, etc.

El acceso a las redes de INFOMED e INDERED, se hará a través del servidor Proxy contando con la implementación de un Firewall.

\section{Filtrado de paquetes}

Tenemos instalado en el servidor Proxy un cortafuego de filtrado de paquetes, que permite pasar los paquetes según sea la dirección de la que vienen, la dirección a la que van, y los puertos respectivos.

Además realiza la traducción de direcciones o enmascaramiento, lo que permite conectar toda una red a Internet empleando una única dirección IP y que no se conozcan las direcciones interiores a la red desde fuera.

\section{Herramienta de administración y monitoreo}

Se cuenta además con el programa LAN Guard Network Scanner, herramienta de seguridad que nos permite chequear los puertos abiertos en cada computadora de la red, el usuario que está conectado, las carpetas que están compartidas en cada computadora y las direcciones IP. Se utiliza el Internet Access Monitor 3.9 c para el análisis de las trazas.4

182 UNESUM-Ciencias.Publicación cuatrimestral. Vol. 5, Año 2021, No. 4 (Número Especial), 


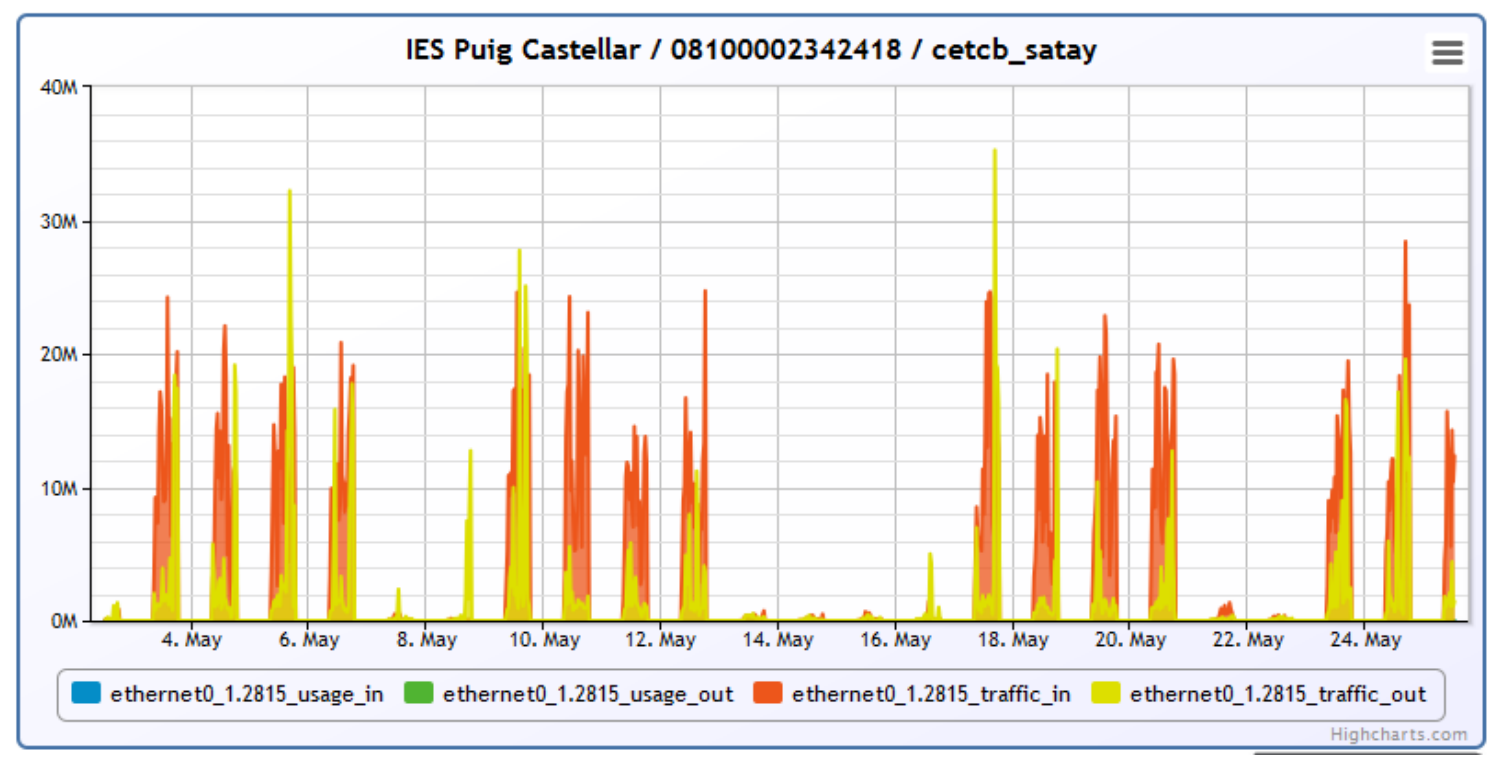

Figura 8. Muestra Trafico Ethernet

\section{CONCLUSIONES}

Esta propuesta centra su investigación en crear una metodología para análisis y diseño de redes, del Instituto de Medicina Deportiva fundamentándose en las prácticas definidas, debido a que las tendencias actuales fijan su orientación en mejorar considerablemente la comunicación entre el área de TI y los clientes.

Como tal existen muchas técnicas diferentes para realizar el análisis y diseño de una red, es importante definir una nueva forma de analizar y diseñar una red, para complementar las técnicas existentes con los requisitos fundamentales de la institución, esto debe ser llevado a cabo con todas las herramientas disponibles.

Diseñar una red de manera adecuada es un reto que involucra algo más que realizar una interconexión física entre dos o más computadores, una red requiere cumplir muchas características para sea escalable y administrable.

Para diseñar una red confiable que cumpla con los requisitos de escalabilidad, confiabilidad y proyección a futuro. 


\section{REFERENCIASBIBLIOGRÁFICAS}

Ávila-Rodríguez, V., Fernández-Ávila, D. G., Muñoz-Velandia, Ó., \& García-Peña, Á. A. (2021). Uso e impacto en las redes sociales por parte de las revistas de reumatología. Revista Colombiana de Reumatología, 28(2), 89-94. https://www.sciencedirect.com/science/article/pii/S0121812320301213

Barba, M. B., Romero, D. G., Fernández, I. F., Ojeda, A. C., Salas, F. M. R., \& Cano, H. T. (2018). Uso de Internet por los adolescentes en la búsqueda de información sanitaria. Atención Primaria, 50(9), 547-552. https://www.sciencedirect.com/science/article/pii/S0212656716306047

Bustos-Claro, M., Ávila, V., Fernández-Ávila, D., Muñoz-Velandia, Ó., \& García, Á. (2021). Relación entre la actividad en las redes sociales de las revistas de medicina interna y las citaciones que reciben. Revista Clínica Española. https://www.sciencedirect.com/science/article/pii/S001425652030299X

Caycho-Rodríguez, T., Ventura-León, J., \& Barboza-Palomino, M. (2021). Diseño y validación de una escala para medir la preocupación por el contagio de la COVID-19 (PRE-COVID-19). Enfermería Clínica, 31(3), 175183. https://www.sciencedirect.com/science/article/pii/S1130862120305350

Cornelio, O. M. (2016). Estación de trabajo para la práctica de Microbiología y Parasitología Médica en la carrera de medicina integrado al sistema de laboratorios a distancia. Revista de Ciencias Médicas de Pinar del Río, 20(2), 174-181.

Cornelio, O. M., \& Gulín, J. G. (2018). Modelo para la evaluación de habilidades profesionales en un Sistema de Laboratorios a Distancia. Revista Científica, 3(33), 1.

Enríquez, D. A. M., Acosta, H. P., Chavira, J. A. P., \& Loya, J. L. (2019). Smartphones y uso de redes sociales: incidencia en el burnout en médicos residentes. Educación Médica. https://www.sciencedirect.com/science/article/pii/S1575181319302001

Hernán-García, M., Lineros-González, C., \& Ruiz-Azarola, A. (2021). Cómo adaptar una investigación cualitativa a $\begin{array}{lllll}\text { contextos de } \quad \text { confinamiento. } & \text { Gaceta }\end{array}$ https://www.sciencedirect.com/science/article/pii/S0213911120301412

Mar-Cornelio, O., Santana-Ching, I., \& González-Gulín, J. (2019). Sistema de Laboratorios Remotos para la práctica de Ingeniería de Control. Revista Científica(36), 356-366.

Mar Cornelio, O., Gulín González, J., Santana Ching, I., \& Rozhnova, L. (2016). Sistema de Laboratorios a Distancia para la práctica de Control Automático. Revista Cubana de Ciencias Informáticas, 10(4), 171183.

Mar, O., Santana, I., \& Gulín, J. (2019). Algorithm to determine and eliminate neutral nodes in Cognitive Neutrosophic Map. Neutrosophic Computing and Machine Learning, 8, 4-11.

Mar, O., Véliz, Y. Z., Felipe, M. d. R. C., \& Vázquez, M. L. (2015). Motor de inferencia decisional en sistema informático para la evaluación del desempeño. Revista Cubana de Ciencias Informáticas, 9(4), 16-29.

Martinez-Perez, C., Alvarez-Peregrina, C., Villa-Collar, C., Arance-Gil, A., \& Sánchez-Tena, M. (2021). Análisis de redes de citación sobre los diversos tipos de lentes intraoculares multifocales. Archivos de la Sociedad Española de Oftalmología. https://www.sciencedirect.com/science/article/pii/S0365669120304925

184 UNESUM-Ciencias.Publicación cuatrimestral. Vol. 5, Año 2021, No. 4 (Número Especial), 